\title{
A generic polynomial solution for the differential equation of hypergeometric type and six sequences of orthogonal polynomials related to it
}

\author{
WOLFRAM KOEPF*† and MOHAMMAD MASJED-JAMEI + \\ $\dagger$ Department of Mathematics, University of Kassel, Heinrich-Plett-Str. 40, D-34132 Kassel, Germany \\ $\$$ Department of Mathematics, K.N. Toosi University of Technology, Sayed Khandan, Jolfa Avenue, \\ Tehran, Iran
}

(Received 6 January 2005)

\begin{abstract}
In this work, we present a generic formula for the polynomial solution families of the well-known differential equation of hypergeometric type

$$
\sigma(x) y_{n}^{\prime \prime}(x)+\tau(x) y_{n}^{\prime}(x)-\lambda_{n} y_{n}(x)=0
$$

and show that all the three classical orthogonal polynomial families as well as three finite orthogonal polynomial families, extracted from this equation, can be identified as special cases of this derived polynomial sequence. Some general properties of this sequence are also given.
\end{abstract}

Keywords: Classical orthogonal polynomials; Differential equation of hypergeometric type; Weight function; Hypergeometric functions; Hypergeometric identities; Recurrence equations

$\operatorname{MSC}(2000): 33 \mathrm{C} 45 ; 33 \mathrm{C} 20 ; 33 \mathrm{~F} 10$

\section{Introduction}

Let us consider the differential equation

$$
\sigma(x) y_{n}^{\prime \prime}(x)+\tau(x) y_{n}^{\prime}(x)-\lambda_{n} y_{n}(x)=0
$$

and search for its polynomial solutions in form $P_{n}(x)=k_{n} x^{n}+k_{n-1} x^{n-1}+\cdots$

We will often consider the monic case $k_{n}=1$. Substituting this form in equation (1) for $n=1$ shows that $\tau(x)=d x+e$ must be a polynomial of degree 1 , and substitution for $n=2$ shows that $\sigma(x)=a x^{2}+b x+c$ is a polynomial of degree at most 2 . Finally, equating the coefficients of $x^{n}$ yields $\lambda_{n}=n(n-1) a+n d$ as the eigenvalue parameter depending on $n=0,1,2, \ldots$

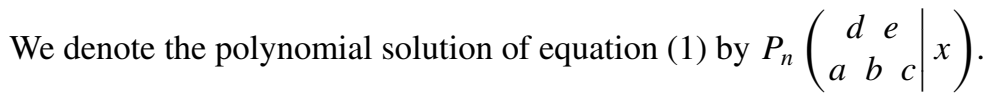

*Corresponding author. Email: koepf@mathematik.uni-kassel.de 
Extensive research has been done on equation (1) and its polynomial solutions up to now. In 1929, Bochner [1] classified the polynomial solutions of equation (1). He showed that the only polynomial systems up to a linear change of variable arising as eigenfunctions of the differential equation (1) are

(i) Jacobi polynomials $\left\{P_{n}^{(\alpha, \beta)}(x)\right\}_{n=0}^{\infty}, \quad(\alpha, \beta, \alpha+\beta+1 \notin\{-1,-2, \ldots\}$,

(ii) Laguerre polynomials $\left\{L_{n}^{(\alpha)}(x)\right\}_{n=0}^{\infty}, \quad(\alpha \notin\{-1,-2, \ldots\}$,

(iii) Hermite polynomials $\left\{H_{n}(x)\right\}_{n=0}^{\infty}$

(iv) Bessel polynomials $\left\{B_{n}^{(\alpha, \beta)}(x)\right\}_{n=0}^{\infty}, \quad(\alpha \notin\{0,-1,-2, \ldots$,$\} and \beta \neq 0)$.

It turns out that, for certain parameter constellations $(\alpha, \beta>-1)$, the first three classes are orthogonal families in a real interval, whereas the fourth class is orthogonal on the unit circle. Nevertheless, for certain parameter instances, there are finite orthogonal families in a real interval in the Bessel case too.

Then, in 1988, Nikiforov and Uvarov [2] gave some general properties of $P_{n}\left(\begin{array}{ccc}d & e \\ a & b & c\end{array} \mid x\right)$, such as a generating function for the polynomials, a Cauchy integral representation etc. in terms of given $\sigma(x)=a x^{2}+b x+c$ and $\tau(x)=d x+e$. Their approach is based on the Rodrigues representation of the polynomials and is not expressed in an explicit form.

Before deriving a generic solution for equation (1), we give an algebraic identity, which is easy to prove but important.

\subsection{An identity}

If $a, b$ and $C_{k}(k=0,1, \ldots, n)$ are real numbers then

$$
\begin{aligned}
\sum_{k=0}^{n} C_{k}(a x+b)^{k} & =\sum_{k=0}^{n}\left(\sum_{i=0}^{n-k}\left(\begin{array}{c}
n-i \\
k
\end{array}\right) b^{n-i} C_{n-i}\right)\left(\frac{a x}{b}\right)^{k} \\
& =\sum_{k=0}^{n}\left(\sum_{i=0}^{k}\left(\begin{array}{c}
n-i \\
k-i
\end{array}\right) b^{k-i} C_{n-i}\right)(a x)^{n-k}
\end{aligned}
$$

For instance, if we set

$$
C_{k}=\frac{(-n)_{k}\left(p_{2}\right)_{k}\left(p_{3}\right)_{k} \cdots\left(p_{m}\right)_{k}}{\left(q_{1}\right)_{k}\left(q_{2}\right)_{k} \cdots\left(q_{m-1}\right)_{k} k !}
$$

such that $(r)_{k}=r(r+1) \cdots(r+k-1)$ denotes the Pochhammer symbol, then we get

$$
\begin{aligned}
{ }_{m} F_{m-1} & \left(\begin{array}{cc}
-n, & p_{2}, \ldots, p_{m} \\
q_{1}, & q_{2}, \ldots, q_{m-1}
\end{array} \mid a x+b\right) \\
= & \frac{(-b)^{n}\left(p_{2}\right)_{n} \cdots\left(p_{m}\right)_{n}}{\left(q_{1}\right)_{n}\left(q_{2}\right)_{n} \cdots\left(q_{m-1}\right)_{n}} \sum_{k=0}^{n}\left(\begin{array}{l}
n \\
k
\end{array}\right) \\
& \times{ }_{m} F_{m-1}\left(\begin{array}{cc}
k-n, & 1-q_{1}-n, \ldots, 1-q_{m-1}-n \\
1-p_{2}-n, & 1-p_{3}-n, \ldots, 1-p_{m}-n
\end{array} \mid \frac{1}{b}\right)\left(\frac{a x}{b}\right)^{k} \\
= & \frac{(-a x)^{n}\left(p_{2}\right)_{n} \cdots\left(p_{m}\right)_{n}}{\left(q_{1}\right)_{n}\left(q_{2}\right)_{n} \cdots\left(q_{m-1}\right)_{n}} \sum_{k=0}^{n}\left(\begin{array}{l}
n \\
k
\end{array}\right) \\
& \times{ }_{m} F_{m-1}\left(\begin{array}{cc}
-k, & 1-q_{1}-n, \ldots, 1-q_{m-1}-n \\
1-p_{2}-n, & 1-p_{3}-n, \ldots, 1-p_{m}-n
\end{array} \mid \frac{1}{b}\right)\left(\frac{b}{a x}\right)^{k}
\end{aligned}
$$


where

$$
{ }_{p} F_{q}\left(\begin{array}{ll}
a_{1}, & a_{2}, \ldots, a_{p} \\
b_{1}, & b_{2}, \ldots, b_{q}
\end{array} \mid x\right)=\sum_{k=0}^{\infty} \frac{\left(a_{1}\right)_{k}\left(a_{2}\right)_{k} \ldots\left(a_{p}\right)_{k}}{\left(b_{1}\right)_{k}\left(b_{2}\right)_{k} \ldots\left(b_{q}\right)_{k}} \frac{x^{k}}{k !}
$$

denotes the generalized hypergeometric function of order $(p, q)$ [3, Chapter 2]. The coefficient of a hypergeometric function is called a hypergeometric term.

Note that, for computing the relations (3), we have generally used the two identities

$$
\left\{\begin{array}{l}
\Gamma(r+k)=\Gamma(r)(r)_{k} \\
\Gamma(r-k)=\frac{\Gamma(r)(-1)^{k}}{(1-r)_{k}}
\end{array} \Longrightarrow(r)_{n-i}=\frac{(-1)^{i}(r)_{n}}{(1-r-n)_{i}} ; \quad r \in \mathbf{R} ; \quad k, n, i \in \mathbf{Z}^{+} .\right.
$$

An interesting case that takes place for equation (3), when $m=2, a=b=1$, is considered. In this case, we have

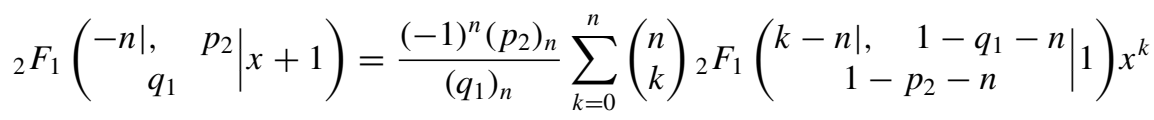

$$
\begin{aligned}
& =\frac{(-x)^{n}\left(p_{2}\right)_{n}}{\left(q_{1}\right)_{n}} \sum_{k=0}^{n}\left(\begin{array}{l}
n \\
k
\end{array}\right){ }_{2} F_{1}\left(\begin{array}{c}
-k \mid, \quad 1-q_{1}-n \\
1-p_{2}-n
\end{array} \mid 1\right) x^{-k},
\end{aligned}
$$

which according to Gauss' identity [3, p. 31]

$$
{ }_{2} F_{1}\left(\begin{array}{cc}
a \mid, & b \\
c & \mid 1
\end{array}\right)=\frac{\Gamma(c) \Gamma(c-a-b)}{\Gamma(c-a) \Gamma(c-b)}
$$

simplifies to

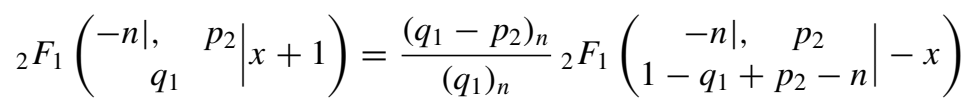

$$
\begin{aligned}
& =\frac{(-x)^{n}\left(p_{2}\right)_{n}}{\left(q_{1}\right)_{n}}{ }_{2} F_{1}\left(\begin{array}{c}
-n \mid, \\
1-p_{1}-n
\end{array} \mid-\frac{1}{x}\right) \text {. }
\end{aligned}
$$

The first identity of equation (7) can be found in ref. [4, (15.3.6)] and the second identity of equation (7) is a special case of ref. [4, (15.3.7)] for integer upper parameter.

Now we are in a good position to state the main theorem of finding a generic polynomial solution for equation (1).

\section{Theorem}

The monic polynomial solution $\bar{P}_{n}\left(\begin{array}{ccc}d & e \\ a & b & c\end{array} \mid x\right)$ of the differential equation

$$
\left(a x^{2}+b x+c\right) y_{n}^{\prime \prime}(x)+(d x+e) y_{n}^{\prime}(x)-n((n-1) a+d) y_{n}(x)=0 ; \quad n \in \mathbf{Z}^{+}
$$

is given by the formula

$$
\bar{P}_{n}\left(\begin{array}{ccc}
d & e \\
a & b & c
\end{array} \mid x\right)=\sum_{k=0}^{n}\left(\begin{array}{l}
n \\
k
\end{array}\right) G_{k}^{(n)}(a, b, c, d, e) x^{k}
$$


where

$$
\begin{aligned}
G_{k}^{(n)}= & \left(\frac{2 a}{b+\sqrt{b^{2}-4 a c}}\right)^{k-n} \\
& \times{ }_{2} F_{1}\left(\begin{array}{c|c}
k-n \mid, \quad \frac{2 a e-b d}{2 a \sqrt{b^{2}-4 a c}}+1-\frac{d}{2 a}-n & \frac{2 \sqrt{b^{2}-4 a c}}{b+\sqrt{b^{2}-4 a c}}
\end{array}\right) .
\end{aligned}
$$

For $a=0$, the equality can be adapted by limit considerations and equation (9) can be given with

$$
G_{k}^{(n)}(0, b, c, d, e)=\lim _{a \rightarrow 0} G_{k}^{(n)}(a, b, c, d, e)=\left(\frac{b}{c}\right){ }_{2}^{k-n} F_{0}\left(k-n, \frac{c d-b e}{b^{2}}+1-n \mid \frac{b^{2}}{c d}\right),
$$

which is valid for $c, d \neq 0$, leading to

$$
\bar{P}_{n}\left(\begin{array}{ccc}
d & e \\
0 & b & c
\end{array} \mid x\right)=\left(\frac{b}{d}\right)^{n}\left(\frac{e b-c d}{b^{2}}\right)_{n} F_{1}\left(\frac{e b-c d}{b^{2}} \mid-\frac{d}{b} x-\frac{c d}{b^{2}}\right) .
$$

For $a=b=0$ and $d \neq 0$, we finally get

$$
\bar{P}_{n}\left(\begin{array}{ccc}
d & e \\
0 & 0 & c
\end{array} \mid x\right)=\lim _{\substack{a \rightarrow 0 \\
b \rightarrow 0}} \bar{P}_{n}\left(\begin{array}{ccc}
d & e \\
a & b & c
\end{array} \mid x\right)=\left(x+\frac{e}{d}\right)^{n}{ }_{2} F_{0}\left(-\frac{n}{2},-\frac{n-1}{2} \mid \frac{2 c d}{(d x+e)^{2}}\right) .
$$

Proof Consider the differential equation (8) and suppose that $x=p t+q$. Therefore, equation (8) changes to

$$
\left(t^{2}+\frac{2 a q+b}{a p} t+\frac{a q^{2}+b q+c}{a p^{2}}\right) \frac{\mathrm{d}^{2} y}{\mathrm{~d} t^{2}}+\left(\frac{d}{a} t+\frac{e+d q}{p a}\right) \frac{\mathrm{d} y}{\mathrm{~d} t}-\frac{n}{a}(d+(n-1) a) y=0 .
$$

Now if, in equation (10), $a q^{2}+b q+c=0$ and $(2 a q+b) / a p=-1$ are assumed, then

$$
p=\mp \frac{\sqrt{b^{2}-4 a c}}{a} \text { and } q=\frac{-b \pm \sqrt{b^{2}-4 a c}}{2 a} .
$$

Thus equation (10) is simplified as

$$
t(t-1) \frac{\mathrm{d}^{2} y}{\mathrm{~d} t^{2}}+\left(\frac{d}{a} t+\frac{2 a e-b d \pm d \sqrt{b^{2}-4 a c}}{\mp 2 a \sqrt{b^{2}-4 a c}}\right) \frac{\mathrm{d} y}{\mathrm{~d} t}-\frac{n}{a}(d+(n-1) a) y=0 .
$$

Equation (12) is a special case of the Gauss hypergeometric differential equation [3, p. 26]:

$$
t(t-1) \frac{\mathrm{d}^{2} y}{\mathrm{~d} t^{2}}+((\alpha+\beta+1) t-\gamma) \frac{\mathrm{d} y}{\mathrm{~d} t}+\alpha \beta y=0
$$

for $\alpha=-n, \beta=n-1+d / a$ and

$$
\gamma=\frac{2 a e-b d \pm d \sqrt{b^{2}-4 a c}}{\mp 2 a \sqrt{b^{2}-4 a c}},
$$




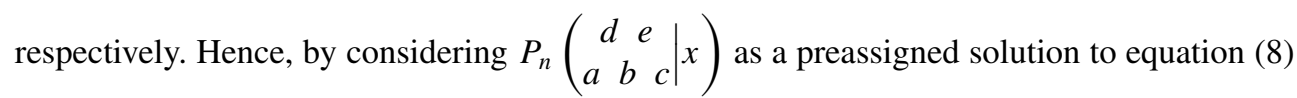
and comparing the relations (12) and (13), we must have

$P_{n}\left(\begin{array}{ccc}d & e \\ a & b & c\end{array} \mid \mp \frac{\sqrt{b^{2}-4 a c}}{a} t+\frac{-b \pm \sqrt{b^{2}-4 a c}}{2 a}\right)=K_{2} F_{1}\left(\begin{array}{c}-n \mid, \quad n-1+d / a \\ \frac{2 a e-b d \pm d \sqrt{b^{2}-4 a c}}{ \pm 2 a \sqrt{b^{2}-4 a c}}\end{array} \mid t\right)$

or written in terms of the variable $x$

$$
P_{n}\left(\begin{array}{ccc}
d & e \\
a & b & c
\end{array} \mid x\right)=K_{2} F_{1}\left(\frac{2 a e-b d \pm d \sqrt{b^{2}-4 a c}}{ \pm 2 a \sqrt{b^{2}-4 a c}} \mid \mp\left(\frac{a x}{\sqrt{b^{2}-4 a c}}+\frac{b \mp \sqrt{b^{2}-4 a c}}{2 \sqrt{b^{2}-4 a c}}\right)\right)
$$

From equation (15), the following sub-cases are obtained

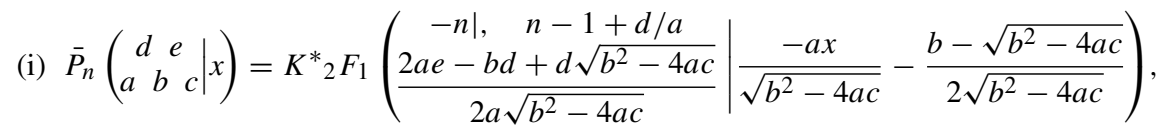

(ii) $\quad \bar{P}_{n}\left(\begin{array}{ccc}d & e \\ a & b & c\end{array} \mid x\right)=K^{* *}{ }_{2} F_{1}\left(\frac{-(2 a e-b d)+d \sqrt{b^{2}-4 a c}}{2 a \sqrt{b^{2}-4 a c}} \mid \frac{a x}{\sqrt{b^{2}-4 a c}}+\frac{b+\sqrt{b^{2}-4 a c}}{2 \sqrt{b^{2}-4 a c}}\right)$.

Note that both the above relations only differ by a minus sign in the argument of the second formula (ii), which does not affect on the differential equation (8).

In other words, if in the first formula (i) we consider the case $\bar{P}_{n}\left(\begin{array}{c}-d-e \\ -a-b-c\end{array} \mid x\right)$, then we will reach the second formula of equation (9). Therefore, only the formula (ii) must be considered as the main solution. To compute $K^{* *}$, it is sufficient to obtain the leading coefficient of ${ }_{2} F_{1}(\cdots \mid \cdots)$ corresponding to formula (ii), which is given by

$$
K^{* *}=\frac{n !\left(\sqrt{b^{2}-4 a c}\right)^{n}\left(\left(b d-2 a e+d \sqrt{b^{2}-4 a c}\right) /\left(2 a \sqrt{b^{2}-4 a c}\right)\right)_{n}}{a^{n}(-n)_{n}(n-1+d / a)_{n}} .
$$

On the other hand, according to identity (3), it can be concluded in general that

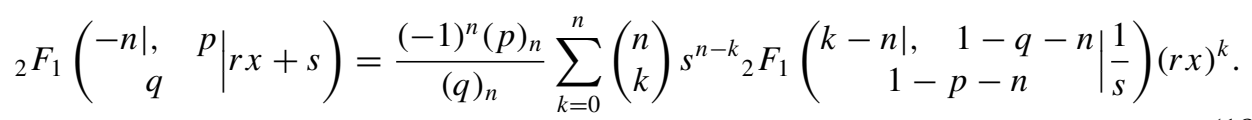

So, by considering the main solution (16) and

$$
\begin{cases}p=n-1+\frac{d}{a}, & q=\frac{b d-2 a e+d \sqrt{b^{2}-4 a c}}{2 a \sqrt{b^{2}-4 a c}} \\ r=\frac{a}{\sqrt{b^{2}-4 a c}}, & s=\frac{b+\sqrt{b^{2}-4 a c}}{2 \sqrt{b^{2}-4 a c}}\end{cases}
$$


equation (18) becomes

$$
\begin{aligned}
{ }_{2} F_{1}\left(\frac{b d-2 a e+d \sqrt{b^{2}-4 a c}}{2 a \sqrt{b^{2}-4 a c}} \mid \frac{a x}{\sqrt{b^{2}-4 a c}}+\frac{b+\sqrt{b^{2}-4 a c}}{2 \sqrt{b^{2}-4 a c}}\right) \\
=\frac{(-1)^{n}((d / a)+n-1)_{n}}{\left(\frac{b d-2 a e+d \sqrt{b^{2}-4 a c}}{2 a \sqrt{b^{2}-4 a c}}\right)_{n}} \times \sum_{k=0}^{n}\left(\begin{array}{l}
n \\
k
\end{array}\right)\left(\frac{b+\sqrt{b^{2}-4 a c}}{2 \sqrt{b^{2}-4 a c}}\right)^{n-k}\left(\frac{a}{\sqrt{b^{2}-4 a c}}\right)^{k} \\
\quad \times{ }_{2} F_{1}\left(\begin{array}{c}
\left.k-n\left|, \quad \frac{2 a e-b d-(d+(2 n-2) a) \sqrt{b^{2}-4 a c}}{2 a \sqrt{b^{2}-4 a c}}\right| \frac{2 \sqrt{b^{2}-4 a c}}{b+\sqrt{b^{2}-4 a c}}\right) x^{k} . \\
-d / a-2 n+2
\end{array}\right.
\end{aligned}
$$

Simplifying this relation and substituting $K^{* *}$ by equation (17), the monic polynomial solution of equation (8) in the form of equation (9) is finally obtained. Hence, the first part of the theorem is proved.

To deduce the limiting case when $a \rightarrow 0$, we have used $\lim _{a \rightarrow 0} a^{r}(-(d / a)+2-2 n)_{r}=$ $(-d)^{r}$ and the following identity

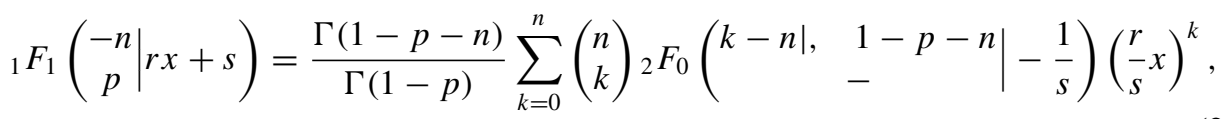

which is a special case of identity (2) for $C_{k}=\left((-n)_{k} /(p)_{k} k !\right)$.

We would like to mention that the general formula $G_{k}^{(n)}(a, b, c, d, e)$ is a suitable tool to compute the coefficients of $x^{k}$ for fixed degree $k$ and arbitrary $a$. If, for example, the coefficient

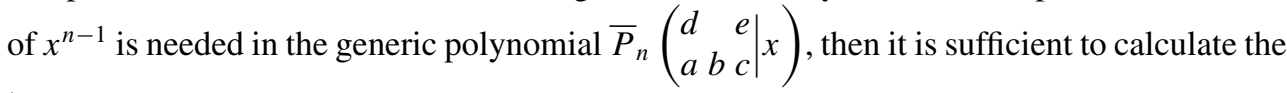
term

$$
\begin{aligned}
& G_{n-1}^{(n)}(a, b, c, d, e)=\left(\frac{2 a}{b+\Delta}\right)^{-1}{ }_{2} F_{1}\left(\begin{array}{ll}
-1 \mid, & \frac{2 a e-b d-(d+(2 n-2) a) \Delta}{2 a \Delta} \\
-\left(\frac{d+(2 n-2) a}{a}\right) & \left.\frac{2 \Delta}{b+\Delta}\right) \\
& =\left(\frac{b+\Delta}{2 a}\right)\left(1+\frac{2 a e-b d-(d+(2 n-2) a) \Delta}{2 a \Delta} \times \frac{2 \Delta}{b+\Delta} \frac{a}{d+(2 n-2) a}\right) \\
& =\frac{e+(n-1) b}{d+(2 n-2) a}
\end{array}\right.
\end{aligned}
$$

in which $\Delta=\sqrt{b^{2}-4 a c}$. Note that in the above simplified relation, all parameters $a, b, c, d$ and $e$ are free and can adopt any value including zero as it is easy to show that neither the values $a$ and $d$ nor the values $b$ and $e$ in equation (9) can vanish together. After simplifying 
$G_{k}^{(n)}(a, b, c, d, e)$ for $k=n-1, n-2, \ldots$, we eventually have

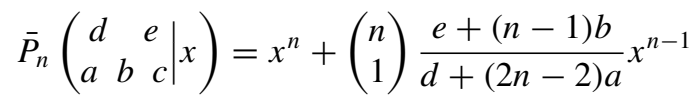

$$
\begin{aligned}
& +\left(\begin{array}{l}
n \\
2
\end{array}\right) \frac{(e+(n-1) b)(e+(n-2) b)+c(d+(2 n-2) a)}{(d+(2 n-2) a)(d+(2 n-3) a)} x^{n-2} \\
& +\cdots+\left(\begin{array}{l}
n \\
n
\end{array}\right)\left(\frac{b+\sqrt{b^{2}-4 a c}}{2 a}\right)^{n} \\
& \times{ }_{2} F_{1}\left(\begin{array}{c|c}
-n & \frac{2 a e-b d-(d+(2 n-2) a) \sqrt{b^{2}-4 a c}}{2 a \sqrt{b^{2}-4 a c}} \\
-\left(\frac{d+(2 n-2) a}{a}\right) & \frac{2 \sqrt{b^{2}-4 a c}}{b+\sqrt{b^{2}-4 a c}}
\end{array}\right) .
\end{aligned}
$$

The above relation implies that

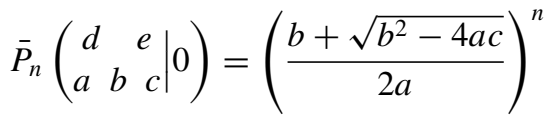

$$
\begin{aligned}
& \times{ }_{2} F_{1}\left(\begin{array}{c|c}
-n & \frac{2 a e-b d-(d+(2 n-2) a) \sqrt{b^{2}-4 a c}}{2 a \sqrt{b^{2}-4 a c}} \\
-\left(\frac{d+(2 n-2) a}{a}\right) & \frac{2 \sqrt{b^{2}-4 a c}}{b+\sqrt{b^{2}-4 a c}}
\end{array}\right) .
\end{aligned}
$$

Also, equation (23) shows, for example, that if $n=0,1,2,3$ then

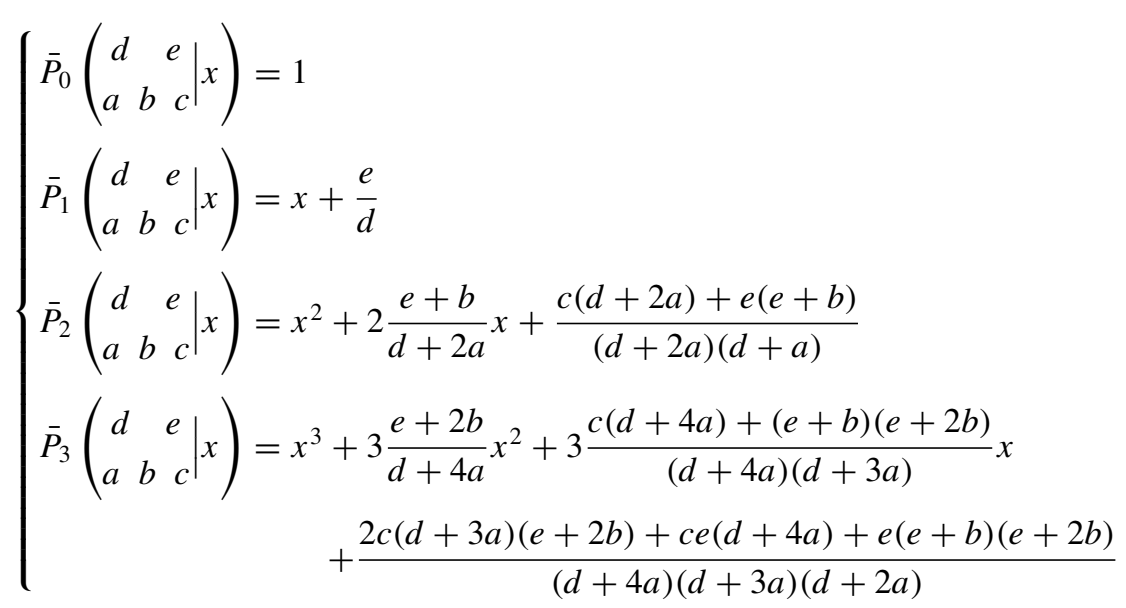

\section{A special case of the generic polynomials (9)}

In the sequel, we would like to apply Gauss' identity, expressed in relation (6). So, let us assume that $\left(2 \sqrt{b^{2}-4 a c} /\left(b+\sqrt{b^{2}-4 a c}\right)\right)=1$ in equation (9), which implies $a c=0$. If $c=0$ 
is supposed, then the following special case for the generic polynomial (9) is derived:

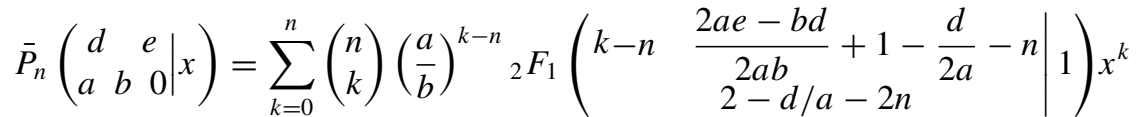

$$
\begin{aligned}
& =\sum_{k=0}^{n}\left(\begin{array}{l}
n \\
k
\end{array}\right)\left(\frac{a}{b}\right)^{k-n} \frac{\Gamma(2-2 n-d / a) \Gamma(1-k-e / b)}{\Gamma(2-n-k-d / a) \Gamma(1-n-e / b)} x^{k} \\
& =\frac{b^{n} \Gamma(2-2 n-d / a) \Gamma(1-e / b)}{a^{n} \Gamma(2-n-d / a) \Gamma(1-n-e / b)} \\
& \times{ }_{2} F_{1}\left(\begin{array}{c}
-n \mid, \quad n-1+d / a \\
e / b
\end{array} \mid-\frac{a}{b} x\right) .
\end{aligned}
$$

On the other hand, let us review the Nikiforov and Uvarov approach and consider the differential equation (8) again. If this equation is written in self-adjoint form, then the weight function $W(x)$ satisfies Pearson's differential equation $(d / d x)(\sigma(x) W(x))=\tau(x) W(x)$ and therefore

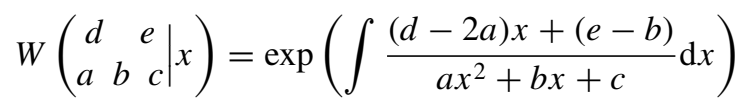

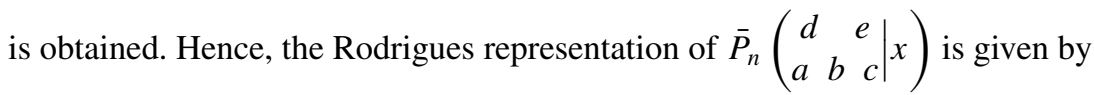

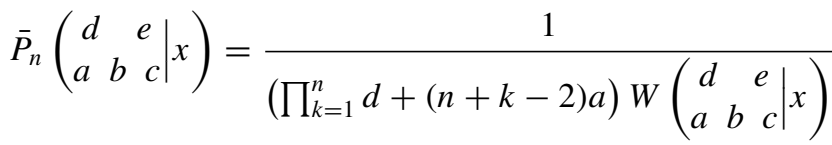

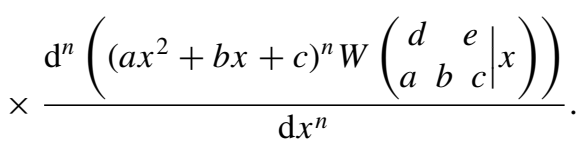

If we put $c=0$ in this relation and use equation (26), we get

$$
\begin{aligned}
\frac{\exp (}{\left.-\int((d-2 a) x+(e-b) \mid) /\left(a x^{2}+b x\right) \mathrm{d} x\right)} \\
\prod_{k=1}^{n} \mid(d+(n+k-2) a) \\
\times \frac{\mathrm{d}^{n}\left(\left(a x^{2}+b x\right)^{n} \exp \left(\int\left((d-2 a) x+(e-b) /\left(a x^{2}+b x\right) \mathrm{d} x\right)\right)\right.}{\mathrm{d} x^{n}} \\
=\frac{\Gamma(2-2 n-d / a)}{\Gamma(1-n-e / b)} \sum_{k=0}^{n}\left(\begin{array}{l}
n \\
k
\end{array}\right)\left(\frac{a}{b}\right)^{k-n} \frac{\Gamma(1-k-e / b)}{\Gamma(2-n-k-d / a)} x^{k} .
\end{aligned}
$$




\section{Some general properties of the main polynomials (9)}

\subsection{A linear change of variables}

Using equation (28), one can derive a linear change of variables for $\bar{P}_{n}\left(\begin{array}{ccc}d & e \\ a & b & c\end{array} \mid x\right)$ explicitly. Assuming $x=w t+v$, the relation (28) changes to

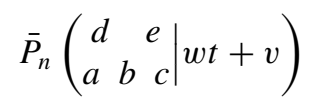

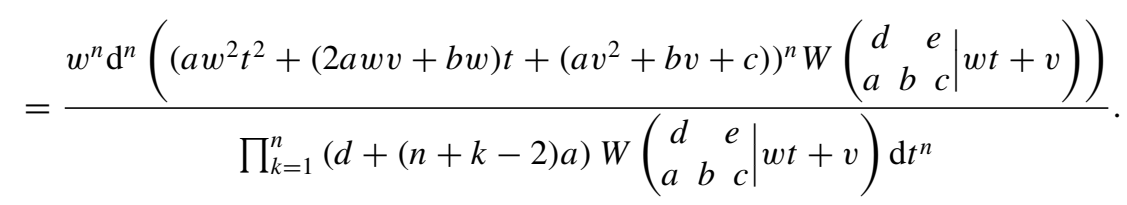

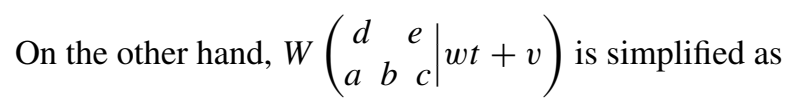

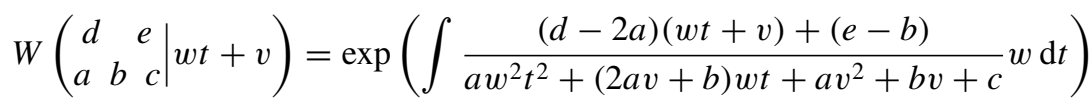

$$
\begin{aligned}
& =W\left(\begin{array}{c}
d w^{2}, \quad(d v+e) w \\
a w^{2},(2 a v+b) w, a v^{2}+b v+c
\end{array} \mid t\right) .
\end{aligned}
$$

Therefore, equation (30) becomes eventually

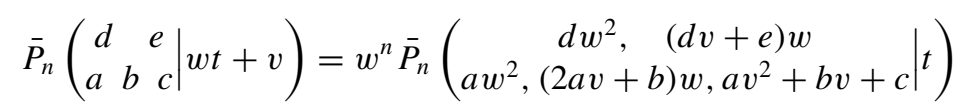

showing the effect of a linear change of variables for the polynomials (9). For instance, if $w=-1$ and $v=0$ are considered, then we have

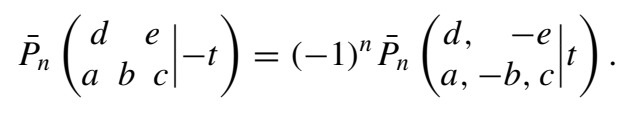

\subsection{A three-term recurrence equation}

The second formula of equation (16) is a proper relation to compute the recurrence equation for the generic polynomials (9). In order to reach this goal, one can use various identities of the Gauss hypergeometric function. For example, the following identity is valid for ${ }_{2} F_{1}(p, q, r ; t)$ [5]:

$$
\begin{aligned}
& (p-q)((p-q-1)(p-q+1) t+2 p q+r(1-p-q)){ }_{2} F_{1}(p, q, r ; t) \\
& \quad+q(r-p)(p-q+1){ }_{2} F_{1}(p-1, q+1, r ; t) \\
& \quad+p(r-q)(p-q-1){ }_{2} F_{1}(p+1, q-1, r ; t)=0 .
\end{aligned}
$$

Now using relation (ii) in equation (16) and its coefficient in equation (17), let us suppose in equation (34) that

$$
\begin{aligned}
& p=-n, \quad q=\frac{d}{a}+n-1, \quad r=\frac{b d-2 a e+d \sqrt{b^{2}-4 a c}}{2 a \sqrt{b^{2}-4 a c}} \text { and } \\
& t=\frac{a}{\sqrt{b^{2}-4 a c}} x+\frac{b+\sqrt{b^{2}-4 a c}}{2 \sqrt{b^{2}-4 a c}} .
\end{aligned}
$$


After some computations, one finally gets

$$
\begin{aligned}
\bar{P}_{n+1}(x)= & \left(x+\frac{2 n(n+1) a b+(d-2 a)(e+2 n b)}{(d+2 n a)(d+(2 n-2) a)}\right) \bar{P}_{n}(x) \\
& n(d+(n-2) a)\left(c(d+(2 n-2) a)^{2}\right. \\
& +\frac{\left.-n b^{2}(d+(n-2) a)+(e-b)(a(e+b)-b d)\right)}{(d+(2 n-3) a)(d+(2 n-2) a)^{2}(d+(2 n-1) a)} \bar{P}_{n-1}(x)
\end{aligned}
$$

in which $\bar{P}_{n}(x)=\bar{P}_{n}\left(\begin{array}{lr}d, & e \\ a, b, c\end{array} \mid x\right)$ and the initial values $\bar{P}_{0}(x)=1$ and $\bar{P}_{1}(x)=x+(e / d)$ are given. For other approaches to deduce equation (36), see refs. [6, 7].

\subsection{The norm square value}

Let $[L, U]$ be the predetermined orthogonality interval, which (besides for finite families, see section 5) of course consists of the zeros of $\sigma(x)=a x^{2}+b x+c$ or $\pm \infty$. Then, by using the Rodrigues representation (28) of the polynomials (9), we have

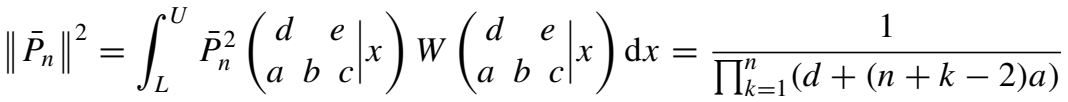

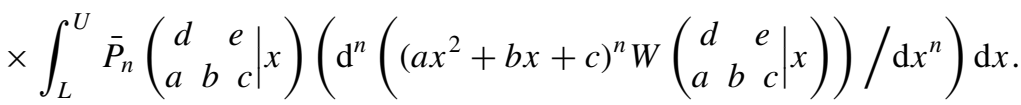

Integration by parts from right hand side of equation (37) eventually yields

$$
\left\|\bar{P}_{n}\right\|^{2}=\frac{n !(-1)^{n}}{\prod_{k=1}^{n} d+(n+k-2) a} \int_{L}^{U}\left(a x^{2}+b x+c\right)^{n}\left(\exp \int \frac{(d-2 a) x+(e-b)}{a x^{2}+b x+c} \mathrm{~d} x\right) \mathrm{d} x .
$$

\section{Six special cases of the generic polynomials (9) as classical orthogonal polynomials}

From the main equation (1), one can extract six special sequences of orthogonal polynomials on the real line. Jacobi, Laguerre and Hermite polynomials are three of them, which nowadays are called the classical orthogonal polynomials. Furthermore, there are three other sequences that are finitely orthogonal for some restricted values of $n$ [8-10]. In this section, we use the previous generic formulas to detect the properties of each of these six sequences.

\subsection{Jacobi orthogonal polynomials}

If $a=-1, b=0, c=1, d=-\alpha-\beta-2$, and $e=-\alpha+\beta$ are selected in equation (9), then

$$
\begin{aligned}
P_{n}^{(\alpha, \beta)}(x) & =\frac{(n+\alpha+\beta+1)_{n}}{2^{n} n !} \bar{P}_{n}\left(\begin{array}{c}
-\alpha-\beta-2,-\alpha+\beta \mid x \\
-1,0,1
\end{array}\right) \\
& \left.=\frac{(n+\alpha+\beta+1)_{n}}{2^{n} n !} \sum_{k=0}^{n}(-1)^{k-n}\left(\begin{array}{l}
n \\
k
\end{array}\right){ }_{2} F_{1}\left(\begin{array}{c}
k-n,-n-\alpha \\
-2 n-\alpha-\beta
\end{array}\right) 2\right) x^{k}
\end{aligned}
$$


are the Jacobi orthogonal polynomials with weight function

$$
W\left(\begin{array}{c}
-\alpha-\beta-2, \quad-\alpha+\beta \\
-1,0,1
\end{array} \mid x\right)=\exp \left(\int \frac{-(\alpha+\beta) x+\beta-\alpha}{-x^{2}+1} \mathrm{~d} x\right)=(1-x)^{\alpha}(1+x)^{\beta}
$$

and orthogonality relation

$$
\begin{aligned}
& \int_{-1}^{1}(1-x)^{\alpha}(1+x)^{\beta} P_{n}^{(\alpha, \beta)}(x) P_{m}^{(\alpha, \beta)}(x) \mathrm{d} x \\
& \quad=\frac{2^{\alpha+\beta+1}}{n !(2 n+\alpha+\beta+1)} \frac{\Gamma(n+\alpha+1) \Gamma(n+\beta+1)}{\Gamma(n+\alpha+\beta+1)} \delta_{n, m},
\end{aligned}
$$

where $\delta_{n, m}=\left\{\begin{array}{ll}0 & \text { if } n \neq m \\ 1 & \text { if } n=m\end{array}\right.$.

The mentioned polynomials can also be represented as

$$
\bar{P}_{n}\left(\begin{array}{c}
-\alpha-\beta-2, \quad-\alpha+\beta \\
-1,0,1
\end{array}\right)=(-1)^{n}(1-x)^{n}{ }_{2} F_{1}\left(\begin{array}{c}
-n,-\alpha-n \\
-\alpha-\beta-2 n
\end{array} \mid \frac{2}{1-x}\right)
$$

which is one of the known hypergeometric representations for the Jacobi polynomials [4, 11].

As the Gegenbauer (ultraspherical), Legendre and Chebyshev polynomials of the first and second kind are all special cases of the Jacobi polynomials, we get the following representations straightforwardly.

Gegenbauer polynomials:

$$
\begin{aligned}
& C_{n}^{(\lambda)}(x)=\frac{2^{n}(\lambda)_{n}}{n !} \bar{P}_{n}\left(\begin{array}{ccc|c}
-2 \lambda & -1 & 0 \\
-1 & 0 & 1 & \mid x
\end{array}\right) \\
& =\frac{2^{n}(\lambda)_{n}}{n !}(-1)^{n}(1-x)^{n}{ }_{2} F_{1}\left(\begin{array}{c|c}
-n, 1 / 2-\lambda-n \\
1-2 \lambda-2 n
\end{array} \mid \frac{2}{1-x}\right),
\end{aligned}
$$

Legendre polynomials:

$$
P_{n}(x)=\frac{(2 n) !}{(n !)^{2} 2^{n}} \bar{P}_{n}\left(\begin{array}{cc|c}
-2 & 0 & x \\
-1 & 0 & 1
\end{array}\right)=\frac{(2 n) !}{(n !)^{2} 2^{n}}(-1)^{n}(1-x)^{n}{ }_{2} F_{1}\left(\begin{array}{c}
-n,-n \\
-2 n
\end{array} \mid \frac{2}{1-x}\right)
$$

Chebyshev polynomials of first kind:

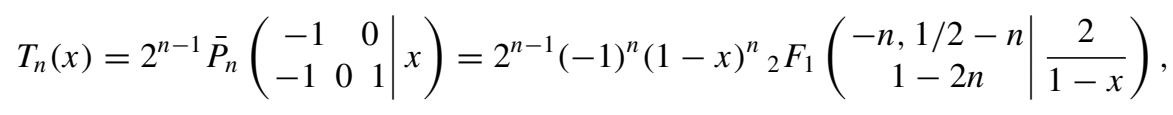

Chebyshev polynomials of second kind:

$$
U_{n}(x)=2^{n} \bar{P}_{n}\left(\begin{array}{ccc}
-3 & 0 & x \\
-1 & 0 & 1
\end{array}\right)=2^{n}(-1)^{n}(1-x)^{n}{ }_{2} F_{1}\left(\begin{array}{c|c}
-n,-1 / 2-n \\
-1-2 n
\end{array} \mid \frac{2}{1-x}\right)
$$




\subsection{Laguerre orthogonal polynomials}

If $a=0, b=1, c=0, d=-1$, and $e=\alpha+1$ are considered in equation (9), then

$$
L_{n}^{(\alpha)}(x)=\frac{(-1)^{n}}{n !} \bar{P}_{n}\left(\begin{array}{rrr}
-1 & \alpha+1 \\
0 & 1 & 0
\end{array} \mid x\right)=\frac{(\alpha+1)_{n}}{n !}{ }_{1} F_{1}\left(\begin{array}{c}
-n \\
\alpha+1
\end{array} \mid x\right)
$$

are the Laguerre orthogonal polynomials with weight function

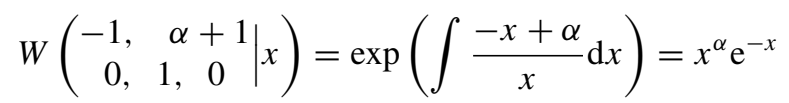

and orthogonality relation

$$
\int_{0}^{\infty} x^{\alpha} \mathrm{e}^{-x} L_{n}^{(\alpha)}(x) L_{m}^{(\alpha)}(x) \mathrm{d} x=\frac{(n+\alpha) !}{n !} \delta_{n, m} .
$$

\subsection{Hermite orthogonal polynomials}

If $a=0, b=0, c=1, d=-2$, and $e=0$ are selected in equation (9), then

$$
H_{n}(x)=2^{n} \bar{P}_{n}\left(\begin{array}{ccc}
-2 & 0 \\
0 & 0 & 1
\end{array} \mid x\right)=(2 x)^{n} \cdot{ }_{2} F_{0}\left(\begin{array}{c}
-\frac{n}{2},-\frac{n-1}{2} \\
-
\end{array} \mid-\frac{1}{x^{2}}\right)
$$

are the Hermite orthogonal polynomials with weight function

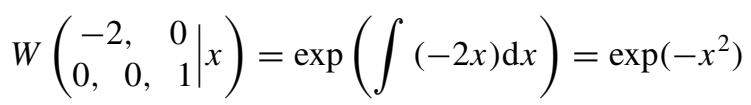

and orthogonality relation

$$
\int_{-\infty}^{\infty} \exp \left(-x^{2}\right) H_{n}(x) H_{m}(x) \mathrm{d} x=n ! 2^{n} \sqrt{\pi} \delta_{n, m} .
$$

\subsection{Finite classical orthogonal polynomials with weight function $W_{1}(x, p, q)=\left(x^{q} /(1+x)^{p+q}\right)$ on $[0, \infty)$}

First let us compute the logarithmic derivative $W_{1}^{\prime}(x) / W_{1}(x)=(-p x+q) /\left(x^{2}+x\right)$. Hence, by Pearson's differential equation or accordingly by equation (27), the main parameters are given as

$$
a=1, b=1, c=0, d=-p+2, e=q+1 .
$$

Note that in ref. [9] the family of polynomials of this type are called Romanovski-Jacobi polynomials, see also ref. [8]. In ref. [10], the related polynomials are denoted by $M_{n}^{(p, q)}(x)$, for which we get

$$
\begin{aligned}
M_{n}^{(p, q)}(x) & =(-1)^{n}(n+1-p)_{n} \bar{P}_{n}\left(\begin{array}{ccc}
-p+2 & q+1 \\
1 & 1 & 0
\end{array} \mid x\right) \\
& =(-1)^{n}(q+1)_{n 2} F_{1}\left(\begin{array}{c}
-n, n+1-p \\
q+1
\end{array} \mid-x\right) .
\end{aligned}
$$

Also, in ref. [10], it is shown that the finite set $\left\{M_{n}^{(p, q)}(x)\right\}_{n=0}^{n=N}$ is orthogonal with respect to the weight function $W_{1}(x, p, q)$ on $[0, \infty)$ if and only if $q>-1$ and $p>2 N+1$. To compute 
the norm square value of the polynomials, we can use the general relation (38). According to this relation, we have

$$
\begin{aligned}
& \int_{0}^{\infty} \frac{x^{q}}{(1+x)^{p+q}} \bar{P}_{n}^{2}\left(\begin{array}{rrr}
-p+2 & q+1 \\
1 & 1 & 0
\end{array} \mid x\right) \mathrm{d} x \\
& \quad=\frac{n !(-1)^{n}}{\prod_{k=1}^{n}(-p+n+k)} \int_{0}^{\infty}\left(x^{2}+x\right)^{n} \frac{x^{q}}{(1+x)^{p+q}} \mathrm{~d} x
\end{aligned}
$$

Then, using equation (53) yields

$$
\int_{0}^{\infty} \frac{x^{q}}{(1+x)^{p+q}}\left(M_{n}^{(p, q)}(x)\right)^{2} \mathrm{~d} x=\frac{n !(p-n-1) !(q+n) !}{(p-2 n-1)(p+q-n-1) !} .
$$

Hence, the complete orthogonality relation is given by

$$
\int_{0}^{\infty} \frac{x^{q}}{(1+x)^{p+q}} M_{n}^{(p, q)}(x) M_{m}^{(p, q)}(x) \mathrm{d} x=\frac{n !(p-n-1) !(q+n) !}{(p-2 n-1)(p+q-n-1) !} \delta_{n, m},
$$

which is valid if and only if $m, n=0,1,2, \ldots, N<(p-1) / 2, q>-1$.

\subsection{Finite classical orthogonal polynomials with weight function $W_{2}(x, p)=x^{-p} \mathrm{e}^{-(1 / x)}$ on $[0, \infty)$}

If we consider the fraction $W_{2}^{\prime}(x) / W_{2}(x)=(-p x+1) / x^{2}$, then the main parameters become

$$
a=1, b=0, c=0, d=-p+2, e=1
$$

In ref. [9], the polynomials of this type are called Romanovski-Bessel polynomials. In ref. [10], these polynomials are denoted by $N_{n}^{(p)}(x)$, for which we have

$$
N_{n}^{(p)}(x)=(-1)^{n}(n+1-p)_{n} \bar{P}_{n}\left(\begin{array}{ccc}
-p+2 & 1 \\
1 & 0 & 0
\end{array} \mid x\right)=(-1)^{n}{ }_{2} F_{0}\left(\begin{array}{c}
-n, 1-p+n \\
-
\end{array} \mid-x\right)
$$

Also, in ref. [10], it is shown that the finite set $\left\{N_{n}^{(p)}(x)\right\}_{n=0}^{n=N}$ is orthogonal with respect to the weight function $W_{2}(x, p)$ on $[0, \infty)$ if and only if $p>2 N+1$. Using the general relation, equation (38) helps us to compute the norm square value of these polynomials as follows:

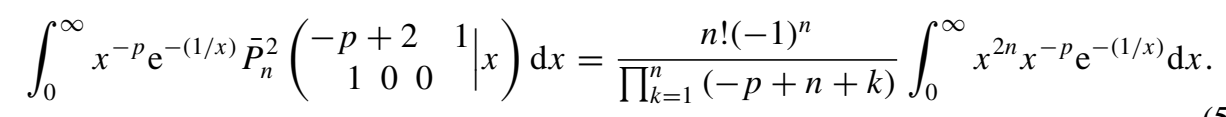

Consequently, the complete orthogonality relation is

$$
\begin{aligned}
& \int_{0}^{\infty} x^{-p} \mathrm{e}^{-(1 / x)} N_{n}^{(p)}(x) N_{m}^{(p)}(x) \mathrm{d} x=\left(\frac{n !(p-(n+1)) !}{p-(2 n+1)}\right) \delta_{n, m} \quad \text { for } \\
& m, n=0,1,2, \ldots, N<\frac{p-1}{2}
\end{aligned}
$$




\subsection{Finite classical orthogonal polynomials with weight function}

$$
\begin{aligned}
& W_{3}^{(p, q)}(x ; A, B, C, D)=\left((A x+B)^{2}+(C x+D)^{2}\right)^{-p} \\
& \exp (q \arctan ((A x+B) /(C x+D))) \text { on }(-\infty, \infty)
\end{aligned}
$$

Similar to the two previous cases, by computing $W_{3}^{\prime}(x) / W_{3}(x)$, we get the parameters

$$
\begin{aligned}
& a=A^{2}+C^{2}, \quad b=2(A B+C D), \quad c=B^{2}+D^{2}, \\
& d=2(1-p)\left(A^{2}+C^{2}\right), \quad e=q(A D-B C)+2(1-p)(A B+C D) .
\end{aligned}
$$

In ref. [9], the polynomials of this type are called Romanovski-pseudo-Jacobi polynomials. In ref. [12], the related polynomials are denoted by $J_{n}^{(p, q)}(x ; A, B, C, D)$, for which we have

$$
\begin{aligned}
J_{n}^{(p, q)} & (x ; A, B, C, D)=(-1)^{n}(n+1-2 p)_{n}\left(A^{2}+C^{2}\right)^{n} \\
& \times \bar{P}_{n}\left(\begin{array}{c}
2(1-p)\left(A^{2}+C^{2}\right), \\
A^{2}+C^{2}, 2(A B D-B C)+2(1-p)(A B+C D)
\end{array} \mid x\right) \\
= & (-1)^{n}(n+1-2 p)_{n}\left(A B+C D+(A D-B C) i+x\left(A^{2}+C^{2}\right)\right)^{n} \\
& \times{ }_{2} \mathrm{~F}_{1}\left(\begin{array}{c}
-n,-n+p-\frac{q}{2} i \\
2 p-2 n
\end{array} \mid \frac{2 i(A D-B C)}{(A-C i)(B+D i+x(A+C i))}\right) .
\end{aligned}
$$

According to ref. [12], these polynomials are finitely orthogonal with respect to the weight function $W_{3}^{(p, q)}(x ; A, B, C, D)$ on $(-\infty, \infty)$ and satisfy the following orthogonality relation:

$$
\begin{aligned}
& \int_{-\infty}^{\infty} W_{3}^{(p, q)}(x ; A, B, C, D) J_{n}^{(p, q)}(x ; A, B, C, D) J_{m}^{(p, q)}(x ; A, B, C, D) \mathrm{d} x \\
& \quad=\left(\frac{n !\left(A^{2}+C^{2}\right)^{n} \Gamma(2 p-n)}{(A D-B C)^{2 p-2 n-1} \Gamma(2 p-2 n)} \int_{-\pi / 2}^{\pi / 2}(A \cos \theta-C \sin \theta)^{2 p-2 n-2} \mathrm{e}^{q \theta} \mathrm{d} \theta\right) \delta_{n, m}
\end{aligned}
$$

valid if and only if $m, n=0,1,2, \ldots, N<p-1 / 2$ and $A D-B C>0, q \in \mathbf{R}$.

\section{How to find the parameters if a special case of the weight function (27) is given?}

By referring to the previous sections, it is easy to see that the best way for finding $a, b, c, d, e$ is to compute the logarithmic derivative $W^{\prime}(x) / W(x)$ and match the pattern in relation (27). Let us clarify this approach by some examples given below.

Example 1 Consider the weight function: $W(x)=\left(-x^{2}+3 x-2\right)^{10} ; 1<x<2$. If the logarithmic derivative of this weight function is computed, then we get

$$
\begin{aligned}
\frac{W^{\prime}(x)}{W(x)} & =\frac{-20 x+30}{-x^{2}+3 x-2}=\frac{(d-2 a) x+(e-b)}{a x^{2}+b x+c} \Longrightarrow \\
a & =-1, b=3, c=-2, d=-22, e=33 .
\end{aligned}
$$

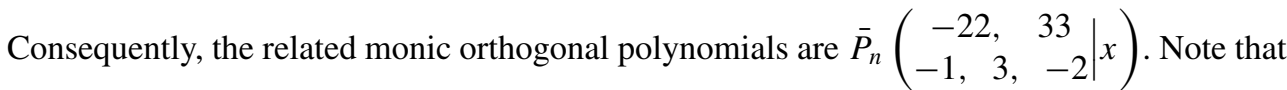
these polynomials are orthogonal with respect to the given weight function on $[1,2]$ for every 
value $n$. Also note that it is not necessary to know that these polynomials are the shifted Jacobi polynomials on the interval $[1,2]$ as they can explicitly and independently be expressed by the generic polynomials (9).

Example 2 If the weight function $W(x)=\left(2 x^{2}+2 x+1\right)^{-10} ;-\infty<x<\infty$ is given, then

$$
\frac{W^{\prime}(x)}{W(x)}=\frac{-40 x-20}{2 x^{2}+2 x+1} \Longrightarrow(a, b, c, d, e)=(2,2,1,-38,-18) \text {. }
$$

Hence, the related monic orthogonal polynomials are $\bar{P}_{n}\left(\begin{array}{cc}-38, & -18 \\ 2, & 2,\end{array} \mid x\right)$. These polynomials are finitely orthogonal for $n \leq 9$, because, according to (63), we must have $N=\max \{n\}<$

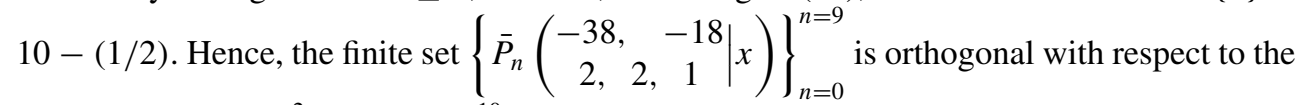
weight function $\left(2 x^{2}+2 x+1\right)^{-10}$ on $(-\infty, \infty)$.

Example 3 Consider the weight function $W(x)=\exp (x(\theta-x)) ;-\infty<x<\infty, \theta \in \mathbf{R}$. Then, we have

$$
\frac{W^{\prime}(x)}{W(x)}=-2 x+\theta \Longrightarrow(a, b, c, d, e)=(0,0,1,-2, \theta)
$$

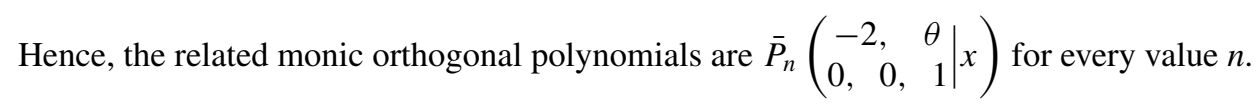

Example 4 For the weight function $W(x)=\left(\sqrt{x} /(x+2)^{8}\right) ; 0<x<\infty$, which is a special case of the second kind of the Beta distribution, we have

$$
\frac{W^{\prime}(x)}{W(x)}=\frac{-15 x+2}{2 x^{2}+4 x} \Longrightarrow(a, b, c, d, e)=(2,4,0,-11,6)
$$

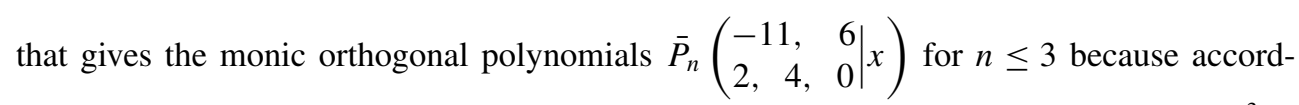

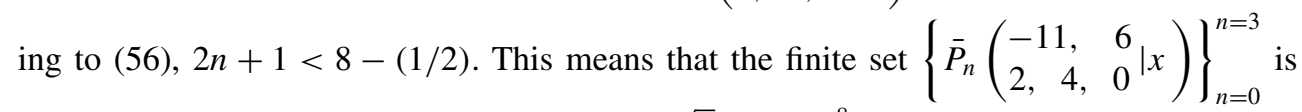
orthogonal with respect to the weight function $\sqrt{x} /(x+2)^{8}$ on $[0, \infty)$.

\section{Classical orthogonal polynomial solutions of three-term recurrence equations}

In ref. [13], this problem was solved in detail. In particular, it was shown how to transform a given three-term recurrence equation into an equivalent three-term recurrence equation for the corresponding monic solution family. Hence, in the sequel, we may assume that the solutions of a given three-term recurrence equation are monic and use the formula (36) derived in this article.

There are two ways to solve the problem to determine the classical orthogonal polynomial solutions of a given three-term recurrence equation. The first way is to compare the given recurrence equation with equation (36). This leads to a system of polynomial equations for the five parameters $a, b, c, d, e$. This procedure was investigated in ref. [13], and a Maple package was developed to perform this task. The second way is to equate the first five terms of each 
two recurrence equations together, which leads to a polynomial system with five equations and five unknowns $a, b, c, d$ and $e$. Let us give an example.

Example 5 If the recurrence equation

$$
\bar{P}_{n+1}(x)=(x+n+3) \bar{P}_{n}(x)-\frac{n(n+1)}{4} \bar{P}_{n-1}(x) ; \quad \bar{P}_{0}(x)=1 \text { and } \bar{P}_{1}(x)=x+3
$$

is given, then find the explicit form of the polynomials, their differential equation, their weight function and finally their orthogonality relation.

Solution If the above recurrence equation is compared with the main equation (36) directly, then one can reach the values $(a, b, c, d, e)=(0,1,2,2,6)$. Therefore, the explicit form of the polynomials are given by $\left.\bar{P}_{n}\left(\begin{array}{cc}2, & 6 \\ 0, & 1,2\end{array}\right)^{x}\right)$ and consequently, their differential equation is found as

$$
(x+2) y_{n}^{\prime \prime}(x)+(2 x+6) y_{n}^{\prime}(x)-2 n y_{n}(x)=0 ; \quad n \in \mathbf{Z}^{+} \text {. }
$$

The above computations are automatically done by the Maple package retode.mpl [13] $>$ read "retode.mpl";

\section{Package “REtoDE”, Maple V-Maple 8}

Copyright 2000-2002, Wolfram Koepf, University of Kassel

$$
\begin{array}{r}
>\mathrm{RE}:=\mathrm{P}(n+1)-(x+n+3) * \mathrm{P}(n)+n *(n+1) / 4 * \mathrm{P}(n-1)=0 \\
\mathrm{RE}:=\mathrm{P}(n+1)-(x+n+3) \mathrm{P}(n)+\frac{1}{4} n(n+1) \mathrm{P}(n-1)=0
\end{array}
$$

$>\operatorname{REtoDE}(\operatorname{subs}(n=n+1, \mathrm{RE}), P(n), x)$;

$$
\begin{aligned}
& {\left[(x+2)\left(\frac{\partial^{2}}{\partial x^{2}} P(n, x)\right)+2(x+3)\left(\frac{\partial}{\partial x} P(n, x)\right)-2 n P(n, x)=0,\right.} \\
& \left.\left[I=[-2, \infty], \rho(x)=\mathrm{e}^{2 x}(x+2)\right], \frac{k_{n+1}}{k_{n}}=1\right]
\end{aligned}
$$

Also, the weight function of these polynomials takes the form

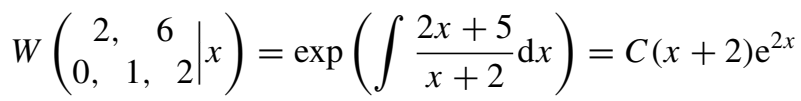

with a constant $C$. Note that the weight function is zero at $x=-2$ and $x=-\infty$. So, the orthogonality interval must be $(-\infty,-2]$. On the other hand, since the weight function must be positive on this interval, $C=-1$, and therefore the weight function is given by $W(x)=-(x+2) \mathrm{e}^{2 x} ;-\infty<x<-2$. Note that the retode program is not set up to decide about the constant $C$ correctly, and hence, wrongly assumed the interval $(-2, \infty]$. Now, 
substituting this weight function in equation (38) yields

$$
\left\|P_{n}\right\|^{2}=\frac{n !(-1)^{n+1}}{2^{n}} \int_{-\infty}^{-2}(x+2)^{n+1} \mathrm{e}^{2 x} \mathrm{~d} x=\frac{\mathrm{e}^{-4} n !(n+1) !}{2^{2 n+2}} .
$$

Finally, the orthogonality relation for the related polynomials will be

$$
\begin{aligned}
& \int_{-\infty}^{-2}\left(-(x+2) \mathrm{e}^{2 x}\right) \bar{P}_{n}\left(\begin{array}{cc|c}
2, & 6 \\
0, & 1, & 2
\end{array} \mid x\right) \bar{P}_{m}\left(\begin{array}{cc|c}
2, & 6 \\
0, & 1, & 2
\end{array} \mid x\right) \mathrm{d} x \\
& =\left(\frac{\mathrm{e}^{-4} n !(n+1) !}{2^{2 n+2}}\right) \delta_{n, m} ; \quad n \in \mathbf{Z}^{+} .
\end{aligned}
$$

Second method If the given recurrence equation is expanded for $n=1,2,3$ and compared with equation (25), then we have the following system:

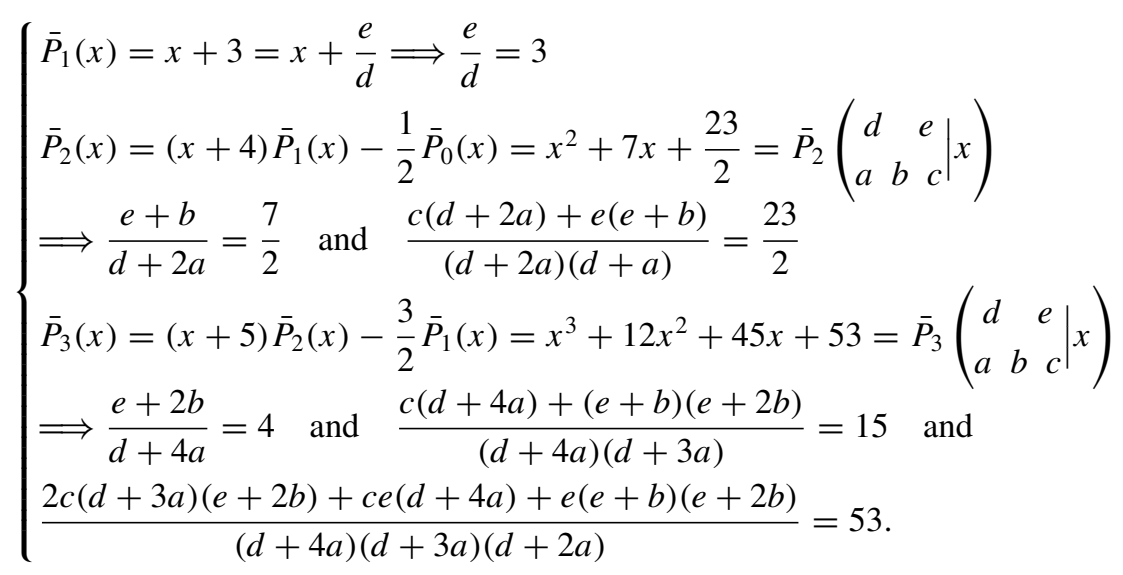

Solving this system eventually yields again that $(a, b, c, d, e)=(0,1,2,2,6)$.

\section{Conclusion}

We have computed a generic formula for the polynomial solution families of the differential equation of hypergeometric type. In particular, our formula gives a unified representation of the classical orthogonal polynomials of Jacobi (including Gegenbauer, Chebyshev and Legendre), Laguerre and Hermite as well as some finite classes of orthogonal polynomials. Furthermore, some applications were given. Using computer algebra, we expect further applications of our result.

The Maple software used in this article (hsum, retode) can be downloaded from the web site http://www.mathematik.uni-kassel.de/ koepf/Publikationen.

\section{References}

[1] Bochner, S., 1929, Über Sturm-Liouvillesche Polynomsysteme. Mathematische Zeitschrift, 29, 730-736.

[2] Nikiforov, A.F. and Uvarov, V.B., 1988, Special Functions of Mathematical Physics (Basel-Boston: Birkhäuser).

[3] Koepf, W., 1988, Hypergeometric Summation (Braunschweig/Wiesbaden: Vieweg).

[4] Abramowitz, M. and Stegun, I.A., 1972, Handbook of Mathematical Functions with Formulas, Graphs, and Mathematical Tables (9th edn) (New York: Dover).

[5] Arfken, G., 1985, Mathematical Methods for Physicists (Academic Press Inc.). 
[6] Lesky, W., 1985, Über Polynomlösungen von Differentialgleichungen und Differen zengleichungen zweiter Ordnung. Anzeigeder-Österreichischen Akademiers der Wissenschaften Mathematisch-Naturwissenchaftliche Klasse, 121, 29-33.

[7] Koepf, W. and Schmersau, D., 1998, Representations of orthogonal polynomials. Journal of Computational and Applied Mathematics, 90, 57-94.

[8] Romanovski, V., 1929, Sur quelques classes nouvelles de polynômes orthogonaux. Comptes Rendues Academie Sciences Paris, 188, 1023-1025.

[9] Lesky, W., 1996, Endliche und unendliche Systeme von kontinuierlichen klassischen Orthogonalpolynomen. Zeitschrift fur Angewandte Mathematik und Mechanik, 76, 181-184.

[10] Masjed-Jamei, M., 2002, Three finite classes of hypergeometric orthogonal polynomials and their application in functions approximation. Journal of Integral Transforms and Special Functions, 13, 169-190.

[11] Szegö, G., 1975, Orthogonal Polynomials (4th edn). (Providence, RI: American Mathematical Society).

[12] Masjed-Jamei, M., 2004, Classical orthogonal polynomials with weight function $\left((a x+b)^{2}+(c x+\right.$ $\left.d)^{2}\right)^{-p} \exp (q \arctan (a x+b) /(c x+d)) ; x \in(-\infty, \infty)$ and a generalization of $T$ and $F$ distributions. Journal of Integral Transforms and Special Functions, 15, 137-153.

[13] Koepf, W. and Schmersau, D., 2002, Recurrence equations and their classical orthogonal polynomial solutions. Applied Mathematics and Computation, 128(2-3), 303-327. 